\title{
Pore Surface Exploration by NMR
}

\section{JH Strange, J Mitchell and JBW Webber}

School of Physical Sciences, University of Kent, Canterbury, Kent, UK, CT2 7NR

Telephone: 01227764000 ext 3271

Fax: 01227827558

E-mail: J.H.Strange@ukc.ac.uk 


\begin{abstract}
A carefully chosen set of experimental techniques applied to porous media characterisation provides results that can be much greater than the sum of the individual parts. The inter-relation and complementarity of a number of techniques will be considered. NMR cryoporometry provides a valuable method of pore size measurement. An NMR method that is more widely used to assess pore dimensions relies on relaxation time analysis of a liquid that fills the pores and the enhanced relaxation that occurs in a liquid at the solid/liquid interface. Thermoporometry, a method based on the application of Differential Scanning Calorimetry (DSC), is closely related to cryoporometry, but employs a different set of assumptions to evaluate pore size distributions. Comparison of the results obtained on the same samples using all these methods together with gas adsorption serves to validate the methods and provide significantly more information about surface-fluid interaction and the behaviour of nano-scale material within pores than each method employed in isolation. Technique developments will be discussed and applications of these methods to ideal silicas will be used to illustrate their power, particularly in combination.
\end{abstract}

\title{
Keywords
}

Porous Media, Nuclear Magnetic Resonance, Cryoporometry, Relaxometry 


\section{Introduction}

There are a variety of methods available for the exploration of porous media, including gas adsorption, mercury porosimetry, neutron scattering, imbibation, DSC (Differential Scanning Calorimetry) thermoporometry and NMR (Nuclear Magnetic Resonance) methods. All of these have advantages and disadvantages and to fully characterise porous media, a range of experiments should be performed using a variety of techniques. These complimentary experiments can provide more information on the pore structure and surface properties than a single technique alone. In this paper we focus on the NMR methods of cryoporometry and relaxometry and their validation and inter-relation using DSC and gas adsorption. Recent development of NMR techniques will be discussed with particular reference to partially filled samples and dual phase systems.

Cryoporometry [1] is a method that utilises the Gibbs-Thomson melting point depression of confined liquids, Eq. (1), where $\Delta T$ is the melting point depression, $x$ is the pore dimension and $k$ is a constant associated with the confined material.

$$
\Delta \mathrm{T}=\frac{\mathrm{k}}{\mathrm{X}}
$$

Cryoporometry is a secondary method in that the melting point depression constant $k$ must first be calibrated for the probe material using samples with known pore size distributions. The solid and liquid NMR signals in the probe material can be distinguished using their different relaxation characteristics resulting in a direct measure of the volume of liquid at any given temperature, thus leading to a pore volume distribution. The solid-liquid transition for material confined within the pores is distinct and easily measured. This method has been well documented for water and cyclohexane [2]. These materials have fixed melting point depression constants of $580 \mathrm{~K} \AA$ and $1790 \mathrm{~K} \AA$ respectively, values that do not change with pore surface structure or porous sample.

Relaxometry uses the enhanced relaxation of molecules at a pore surface to calculate the pore diameter [3] and normally assumes rapid exchange between molecules at the surface and in the pores. The inverse spin-lattice and spin-spin relaxation rates $\left(1 / \mathrm{T}_{1}\right.$ 
and $1 / \mathrm{T}_{2}$ ) are then proportional to the surface $(\mathrm{S})$ to volume $(\mathrm{V})$ ratio [4] of the porous media, as in Eq. (2).

$$
\frac{1}{T_{1}}=\rho_{1} \frac{S}{V} \text { or } \frac{1}{T_{2}}=\rho_{2} \frac{S}{V}
$$

By fitting a distribution of relaxation times to the measured relaxation data a pore size distribution can be estimated. Unfortunately this method is susceptible to noise artefacts [5] and the distributions produced are generally broadened and difficult to compare to those produced by other methods. The relaxivity constant $\rho$, see Eq. (2), unlike the $k$ value in Eq. (1) as used in Cryoporometry or Thermoporometry [6], is liable to change with pore surface composition [7]. We have demonstrated this even for apparently similar sol-gel silicas (see below). Relaxometry has found extensive use in oil-well logging [8]; the rocks having naturally broad pore distributions and the physical constraints of the bore holes prevent any other types of pore size measurement. Although there is some debate over the accuracy of Relaxometry [5], it is an invaluable tool outside the lab environment where other techniques are unavailable. Other NMR experiments for studying interactions at the pore surface include partial filling of pores with liquid [9]. A further adaptation to these methods is to use two immiscible fluids in the pores at the same time [10], providing information on different surface affinities, phase separation and the nature of the internal pore structure. The methods of cryoporometry and relaxometry can be combined [11] to show the change in relaxation times throughout the sample as the confined material melts. This can provide more information on the behaviour of the probe material in the porous system than either of the two methods separately and will be discussed later.

\section{Experimental Considerations}

We now consider additions to the methods and interpretation of NMR cryoporometry. Cryoporometry has been previously shown to produce accurate, repeatable pore size distributions using probe materials with different surface interactions and sub-ambient melting points [2]. A novel modification to this technique is the use of naphthalene as a probe material with a super-ambient melting point $\left(\mathrm{mp} 80.5^{\circ} \mathrm{C}\right)$. This material obeys the Gibbs-Thomson equation and has a large melting point depression constant of 
1810K $\AA$. High-temperature cryoporometry has been compared with gas adsorption and DSC measurements and found to be consistent as can be seen in Fig 1a. Cryoporometry is very similar to DSC thermoporometry [12] in that they both rely on melting point suppression and produce comparable pore size distributions, Fig 1b. In cryoporometry it is necessary to assume the density of the liquid does not change significantly over the temperature range. Cryoporometry is a more direct measurement, the signal amplitude being directly proportional to the volume of liquid in the pores. Cryoporometry is also a static measurement, the temperature being constant whilst the NMR signal is recorded. In comparison, DSC is a dynamic process relying on rate of change to correctly analyse the sample [12]. Whilst DSC has the advantage of speed, NMR cryoporometry has the advantage of producing more detailed and direct volumetric pore size distributions.

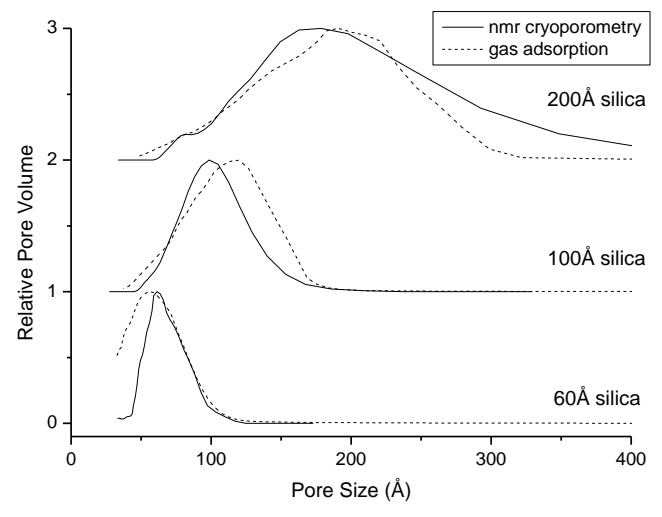

(a)



(b)

Fig 1. Comparison between pore size distributions generated by (a) NMR Cryoporometry and Gas Adsorption and (b) NMR Cryoporometry and DSC Thermoporometry, using naphthalene as the probe.

Two ways of capturing the cryoporometry data have been explored. The first is to ramp the sample temperature and capture multiple NMR spin echoes, each at a slightly different temperature. This produces a large number of data points, each with a significant associated noise level and provides a highly detailed pore volume distribution dependent on signal filtering, e.g. by the application of a smoothing function, so care must be taken not to over interpret the results. The second method is to step the temperature by a fixed amount and capture an entire CPMG (Carr-Purcell Meiboom-Gill) decay curve. This provides a fewer number of points but each point has a lower associated noise when repeated CPMG scans are averaged. 
An important issue in the cryoporometry measurement is the practical consideration of the $\mathrm{T}_{2}$ modification due to surface interactions. The 'ideal' cryoporometric experiment assumes a long $\mathrm{T}_{2}$ in the liquid and a vanishingly small $\mathrm{T}_{2}$ in the solid. In cyclohexane, for example, this is not necessarily the case. In most materials $T_{2}$ of a liquid confined within pores is shorter than the bulk due to surface relaxation. The phase diagram of cyclohexane in a confined solid is complex [13] and leads to a complex transverse relaxation behaviour within the pores. Even in the bulk material $\mathrm{T}_{2}$ of the solid lengthens as it approaches the melting point and this is exaggerated for the material in the pores. This results in an apparent change in the $k$ value for echo measurement times, $2 \tau$, that are on a time scale approaching that of the solid phase $\mathrm{T}_{2}$, see Fig 2b [2]. If measurements are taken at too short a time $\tau$ then the solid state and the liquid state are poorly distinguished, increasing the apparent volume of the large pores at the expense of the small pores in the distribution. This may be corrected if measurements are taken at more than one $\tau$ value [2]. If measurements employ too long a value of $\tau$, then the smaller pores are lost. A more ideal situation arises for the proton NMR signal from the liquid component of naphthalene in $\mathrm{SiO}_{2}$ that shows only one apparent $T_{2}$ in pores with a diameter greater than $60 \AA$. Its value within the pores is at least two orders of magnitude less than that for the bulk liquid, although it changes significantly with temperature and pore geometry. Any measured cryoporometry data set is therefore potentially dependent on the time of the experiment. This is illustrated in Fig $\mathbf{2 a}$ where $\mathrm{T}_{2}$ decay curves (CPMG) for the liquid component of the proton signal from naphthalene are shown at a series of temperatures. However, provided the echo time $(2 \tau)$ is less than $100 \mathrm{~ms}$, the cryoporometry melting curves for naphthalene are independent of $\tau$. 


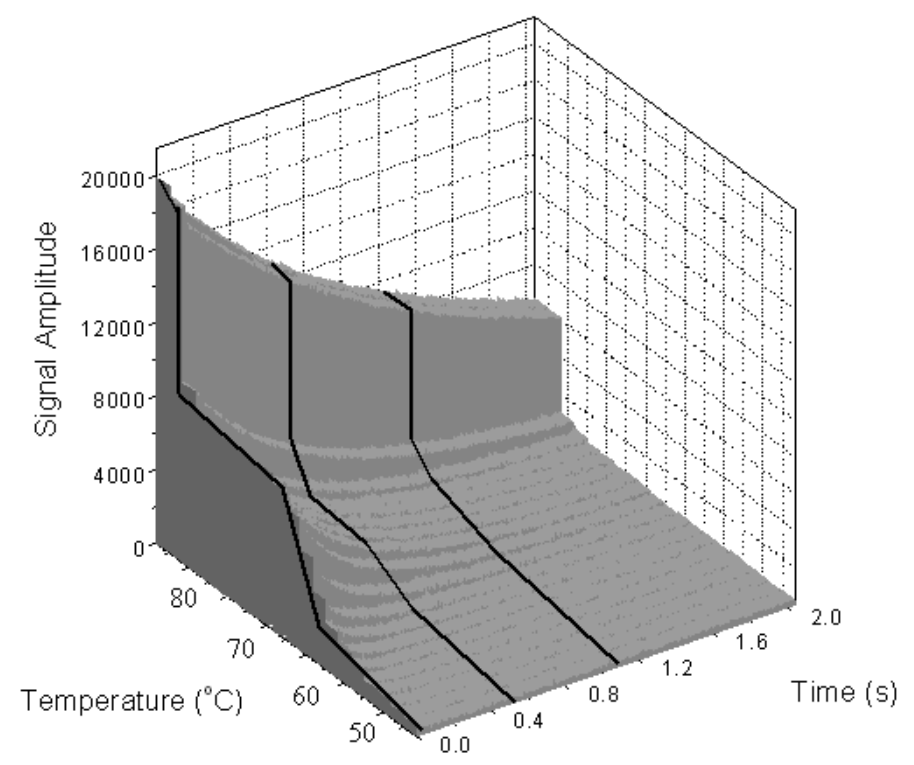

Fig 2(a)

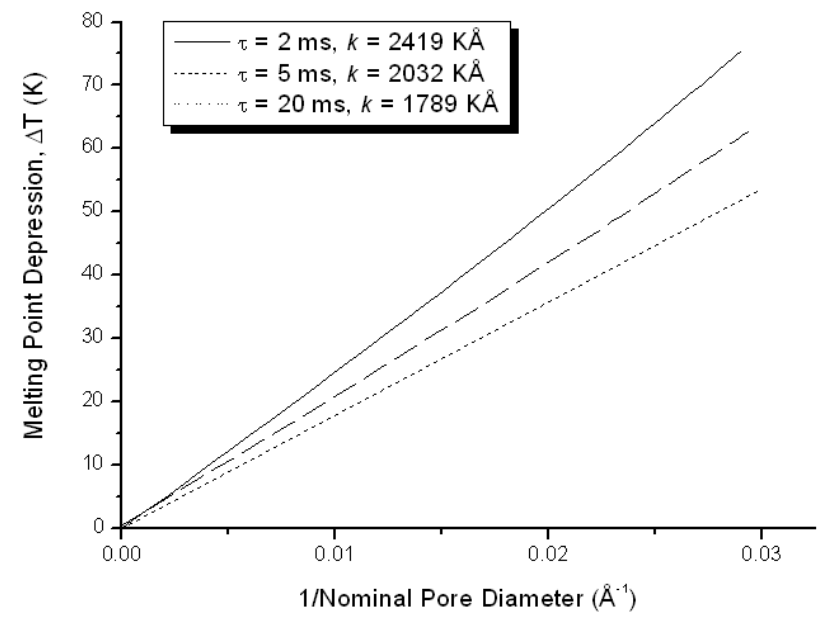

Fig 2(b)

Fig 2a. Transverse magnetisation decay of the liquid component of naphthalene in silica pores of mean diameter $100 \AA$ as a function of temperature. The heavy black lines represent the measured cryoporometry curves at different spin echo times

Fig 2b. Apparent variation of Gibbs-Thomson $k$ constant for cyclohexane as a function of measured echo time $2 \tau$. Some curvature is observed on the upper lines due to surface effects. 


\section{NMR Relaxometry}

By measuring the $\mathrm{T}_{2}$ dephasing of spins in a liquid confined in mono-dispersed solgel silicas, it is possible to estimate a value for the surface relaxivity, knowing the surface-to-volume ratio of the samples from gas adsorption measurements. It was found that silica gels from the same manufacturer could be described by the same surface relaxometry parameter, but the silicas from two different manufacturers gave distinct surface relaxivity values. All samples had similar surface-to-volume ratios. Experiments employed contrasting liquids of water and naphthalene. For water, expected to interact strongly with the surface, the surface relaxivity $\rho$ was found to be $144 \AA \mathrm{s}^{-1}$ for Unilever sorbsil silicas and $66 \AA \mathrm{s}^{-1}$ for Merck silica-gels, both silica samples being manufactured by a sol-gel process (Fig 3). The less surface interactive naphthalene gave much lower relaxivity values of $6.7 \AA^{-1}$ for the Unilever silicas and $2.8 \AA \mathrm{s}^{-1}$ for the Merck silicas. By repeating the experiments with non-polar naphthalene, we show a difference between the surface interactions of the two species of probe material. Relaxometry has been previously used to show that polar molecules behave quite differently in porous media [14]. They interact with the surface $\mathrm{OH}$ groups, greatly increasing their surface residence time. Non-polar molecules interact weakly with the surface and are less susceptible to surface modifications. However, it has also been shown that paramagnetic impurities in the porous media often influence the relaxation times of the fluid [15], modifying the relaxation times which then depend on molecular mobility and concentration of paramagnetic impurities.
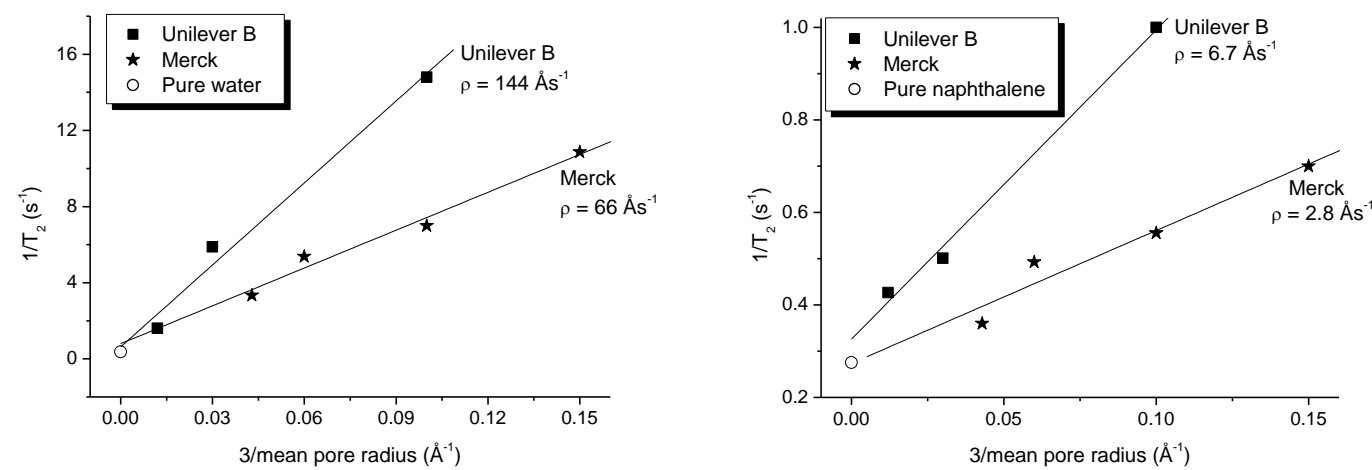

Fig 3. Surface relaxation in Sol-Gel Silicas. Two distinct surface relaxation values have been observed for mono-dispersed sol-gel silicas provided by two different manufacturers for both samples of water and naphthalene. 


\section{Combined Techniques}

The relaxation time for a confined liquid essentially sees an average for a molecule over the time of the experiment. Molecules will therefore travel a distance of microns in a typical relaxation time and see an average pore dimension over this distance [16]. For a partially frozen sample where material in the larger pores is frozen the molecules in the remaining liquid should have a shortened relaxation time corresponding to the smaller pores they experience. If molecules can visit the pore size distribution during this time a simple average will be seen. If molecules are confined each to its own pore sizes on a local scale, a broader distribution will result. By combining cryoporometry and relaxation measurements it is therefore possible to explore the interconnection of the pore size distribution. This has been demonstrated for silica gels samples and also shown to hold promise in its application to cement and mortar hydration by Valckenborg et al [11].

By combining cryoporometry and relaxometry on partially filled samples it has been shown that, at low filling factors in porous silica, water (polar) puddles in surface irregularities whilst cyclohexane (non-polar) completely fills only the smallest pores $[9,17]$. This is an important step in understanding the full picture on the surface interactions between the confined material and the pore surface. The technique can lead to mapping of the wettability (hydrophilicity) in the porous sample, since the spatial location of the absorbed water can be observed. A further possibility is to determine the surface roughness from the size of the water droplets that collect on the pore surface. Beyond this, it should be possible by combining immiscible fluids within pores, to determine different surface affinities and phase separations. This area has yet to be explored fully by NMR and at this time shows considerable promise for development. Experiments are being conducted to see if water and cyclohexane, when mixed in porous silica, behave as each would in an individual partially filled sample. Resultant information would be of importance for understanding catalytic behaviour and processes within chemical reactors. The phase separation of a hexane and nitrobenzene mixture has already been studied by cryoporometry [10] within porous glasses. This work demonstrated the formation of nitrobenzene droplets surrounded by hexane within the porous structure.

NMR imaging techniques can be added to provide a 1, 2 or 3-dimensional representation of the liquid distribution, pore size distribution and fluid interaction in a sample [18]. 


\section{Further Experimentation}

Recently a quenching experiment on cyclohexane in porous silica was undertaken to investigate nucleation rates and metastable states using DSC. It has already been shown that the formation of crystal lattices is impaired within a porous matrix, with the presence of a defective surface layer, estimated to be of the order of two molecular layers in thickness $[13,14]$. The confined liquid cannot form a lattice structure due to the proximity of the surface. By quenching a sample like cyclohexane it is possible to freeze it in a defective lattice state, forming metastable constructs that realign on warming. Due to the extremely small size of the DSC samples (unlike NMR samples), it is easy to quench them. Cyclohexane has a brittle to plastic crystal transition normally occurring at $193 \mathrm{~K}$ and a plastic to liquid transition at $279.5 \mathrm{~K}$. A small sample was rapidly cooled to below $100 \mathrm{~K}$. Three DSC measurement runs were then made on the quenched sample (see Fig 4) when unusual exothermic features were observed [19]. The normal DSC curves show endotherms for the confined material at $-100^{\circ} \mathrm{C}$ and for the bulk at $-85^{\circ} \mathrm{C}$ (brittle to plastic transition) and second endotherms at $-10^{\circ} \mathrm{C}$ (confined) and $+6.5^{\circ} \mathrm{C}$ (bulk) corresponding to the melting transition (cooling curve and second warming curve). Fig 4 also shows that on the first warming curve additional exothermic changes are seen at $-130^{\circ} \mathrm{C}$ and $-18^{\circ} \mathrm{C}$. Fig 4 shows a complete experimental cycle consisting of warming the quenched sample followed by a second controlled cooling and subsequent warming. The cooling scan shows additional super-cooling of bulk and confined material and in the second warming scan the expected solid-solid and solid-liquid endothermic transitions are observed without exothermic anomalies. Upon quenching the liquid sample in the pores, it cools so rapidly that it can enter metastable, partially ordered states that only revert to an ordered structure on warming. The pore system produces isolated nucleation sites allowing for the unusual behaviour not observable in the bulk. The thermal anomalies are attributed to the re-ordering of these states as the temperature rises. NMR relaxation time measurements are in progress to characterise more completely these metastable states. 


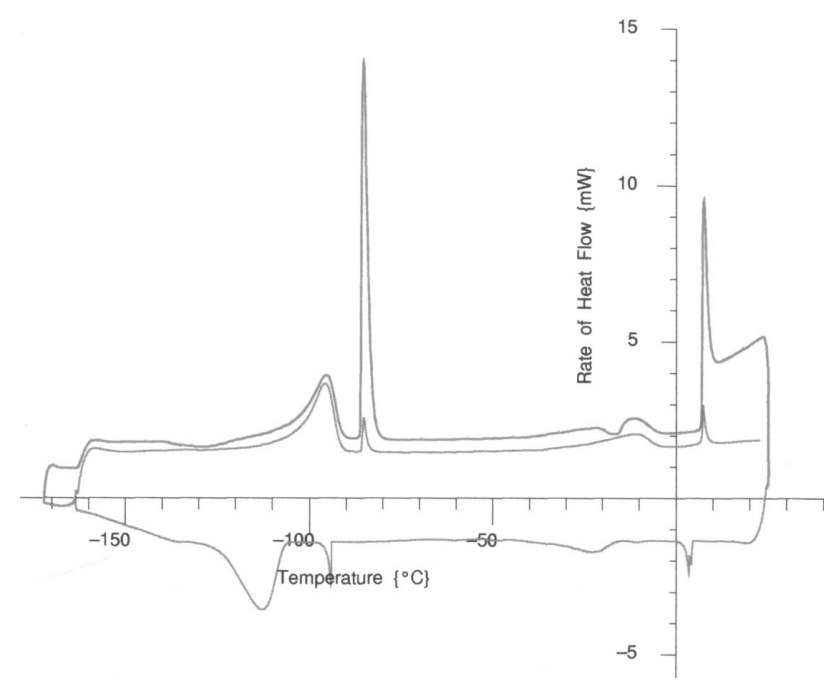

Fig 4. DSC quenching experiment. This plot shows the warming of cyclohexane in $100 \AA$ porous silica after quenching (top line) and after regular cooling (middle line). The quenched warming data clearly shows additional phase transition information corresponding to temperature dependant metastable states.

\section{Conclusions}

NMR cryoporometry has been demonstrated and validated against both gas adsorption and DSC. We have also shown that naphthalene is both reliable and convenient for pore size distribution measurements removing the need for cryogens. Refinements to the method of NMR cryoporometry have also been presented, and the advantages of recording the complete Carr-Purcell Meiboom-Gill (CPMG) echo envelope as a function of temperature are clear. This approach allows for the differing decays in transverse magnetisation to be assessed, and artefacts introduced through the change in echo envelope as a function of temperature and pore geometry [2] to be overcome. NMR cryoporometry, although a secondary method of pore size distribution analysis, appears to be independent of surface morphology for saturated samples. In contrast, NMR relaxometry suffers from problems arising from surface dependent relaxation. Considerable variations in measured transverse relaxation have been observed in apparently similar porous sol-gel silica samples that show little or no difference in pore morphology when measured with other methods. However, by combining these differences in relaxation with knowledge on the concentration of relaxation centres it is possible to extract information on the relative molecular mobility of the liquid and the wettablility of the surface. Using a non-surface-relaxation-sensitive method of determining pore geometry, combined with a surface sensitive method, it is possible 
to deduce the fluid behaviour on the surface. Further to this, combining non-NMR based pore size analysis with NMR measurements as a function of pore filling [17], wettabililty, surface interactions and pore morphology can all be studied. It has been shown that information on surface-fluid interaction within the pores can be obtained and the very different nano-scale behaviour of water and cyclohexane has been observed.

Any additional information that can be obtained about the pore volume/surface structural relations is indispensable. A very powerful source of independent information on this is Small Angle Neutron Scattering (SANS), as the measured scattering results from where there are pore-wall to void interfaces; there is of course no net scattering variation for uniform solids or voids. SANS has been used to successfully study porous silicas in the pore diameter range $2.5 \mathrm{~nm}$ to $50 \mathrm{~nm}$. By a combination of NMR cryoporometry, gas adsorption and imbibation measurements on the same set of silicas and using analytic and numerical models [20], it is possible to provide extensive information on the samples that far exceeds that available from any of the individual methods. NMR is a very powerful probe for porous media and a combination of techniques should be employed for materials characterisation wherever possible.

\section{References}

1. Strange JH, Rahman M, Smith EG. Characterisation of Porous Solids by NMR. Physical Review Letters 1993;71(21):3589-3591.

2. Webber JBW. Characterising Porous Media. PhD Thesis: University of Kent; 2000.

3. Gallegos DP, Munn K, Smith DM, Stermer DL. A NMR technique for the Analysis of Pore Structure: Application to Materials with Well-Defined Pore Structure. Journal of Colloid and Interface Science 1986;119(1):127-140.

4. Cohen MH, Mendelson KS. Nuclear magnetic relaxation and the internal geometry of sedimentary rocks. Journal of Applied Physics 1982;53(2):11271135.

5. Brown RJS, Borgia GC, Fantazzini P, Mesini E. Problems in identifying multimodal distributions of relaxation times for NMR in porous media. Magnetic Resonance Imaging 1991;9:687-693.

6. Jackson CL, McKenna GB. The Melting Behaviour of Organic Materials Confined in Porous Solids. J. Chem. Phys. 1990;93(12):9002-9011.

7. Kleinberg RL. Utility of NMR T2 distributions, connection with capillary pressure, clay effect, and determination of the surface relaxivity parameter $\mathrm{p} 2$. Magnetic Resonance Imaging 1996;14(7/8):761-767. 
8. Kleinberg RL, Kenyon WE, Mitra PP. Mechanism of NMR Relaxation of Fluids in Rock. Journal of Magnetic Resonance 1993;108(Series A):206-214.

9. Allen SG, Stephenson PCL, Strange JH. Internal Surfaces of Porous Media studied by NMR cryoporometry. Chem. Phys. 1998;108(19):8195-8198.

10. Valiullin R, Furo I. Phase separation of a binary liquid mixture in porous media studied by nuclear magnetic resonance cryoporometry. Journal of Chemical Physics 2002;116(3):1072-1076.

11. Valckenborg RME, Pel L, Kopinga K. Combined NMR cryoporometry and relaxometry. Journal of Physics D: Applied Physics 2002;35:249-256.

12. Ishikiriyama K, Todoki M. Pore Size Distribution Measurements of Silica Gels by DSC: Thermoporosimetry. Colloid and Interface Science 1995; 171:103-111.

13. Booth HF, Strange JH. Organic nanocrystals: an NMR study of cyclohexane in porous silica. Molecular Physics 1997;93(2):263-269.

14. Zavada T, Stapf S, Kimmich R. Diffusion and Relaxation in Interface LAyers of Crystals in Nanoporous Glass. Magnetic Resonance Imaging 1998;16(5/6):695-697.

15. Korb JP. Surface dynamics of liquids in porous media. Magnetic Resonance Imaging 2001;19(3-4):363-368.

16. Halperin WP, Jehng JY, Song Y. Application of spin-spin relaxation to measurement of surface area and pore size distributions in a hydrating cement paste. Magnetic Resonance Imaging 1994;12(2):169-173.

17. Allen SG, Stephenson PCL, Strange JH. Morphology of porous media studied by NMR. Chem. Phys. 1997;106(18):7802-7809.

18. Strange JH, Webber JBW. Spatially resolved pore size distributions by NMR. Meas. Sci. Technol. 1997;8(1-7):555-561.

19. Dore J, Webber JBW, Strange JH, Farman H, Descamps M, Carpentier L. Phase Transformations for Cyclohexane in Mesoporous Silicas. Physica A 2002:IN PRESS.

20. Webber JBW, Strange JH, Dore JC. An evaluation of NMR Cryoporometry, Density Measurement and Neutron Scattering methods of pore characterisation. Magnetic Resonance Imaging 2001;19(3-4):395-399. 


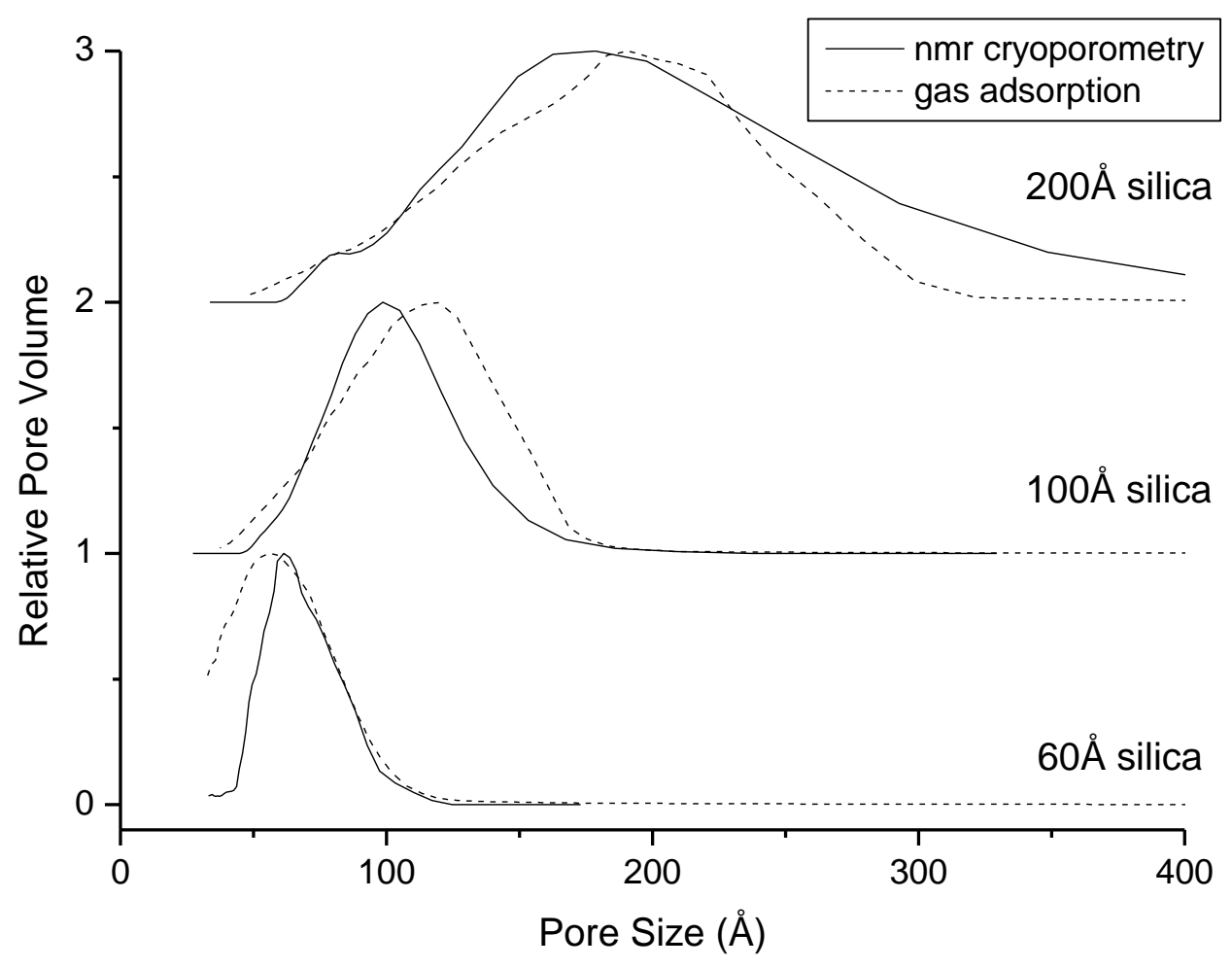

Fig 1(a)

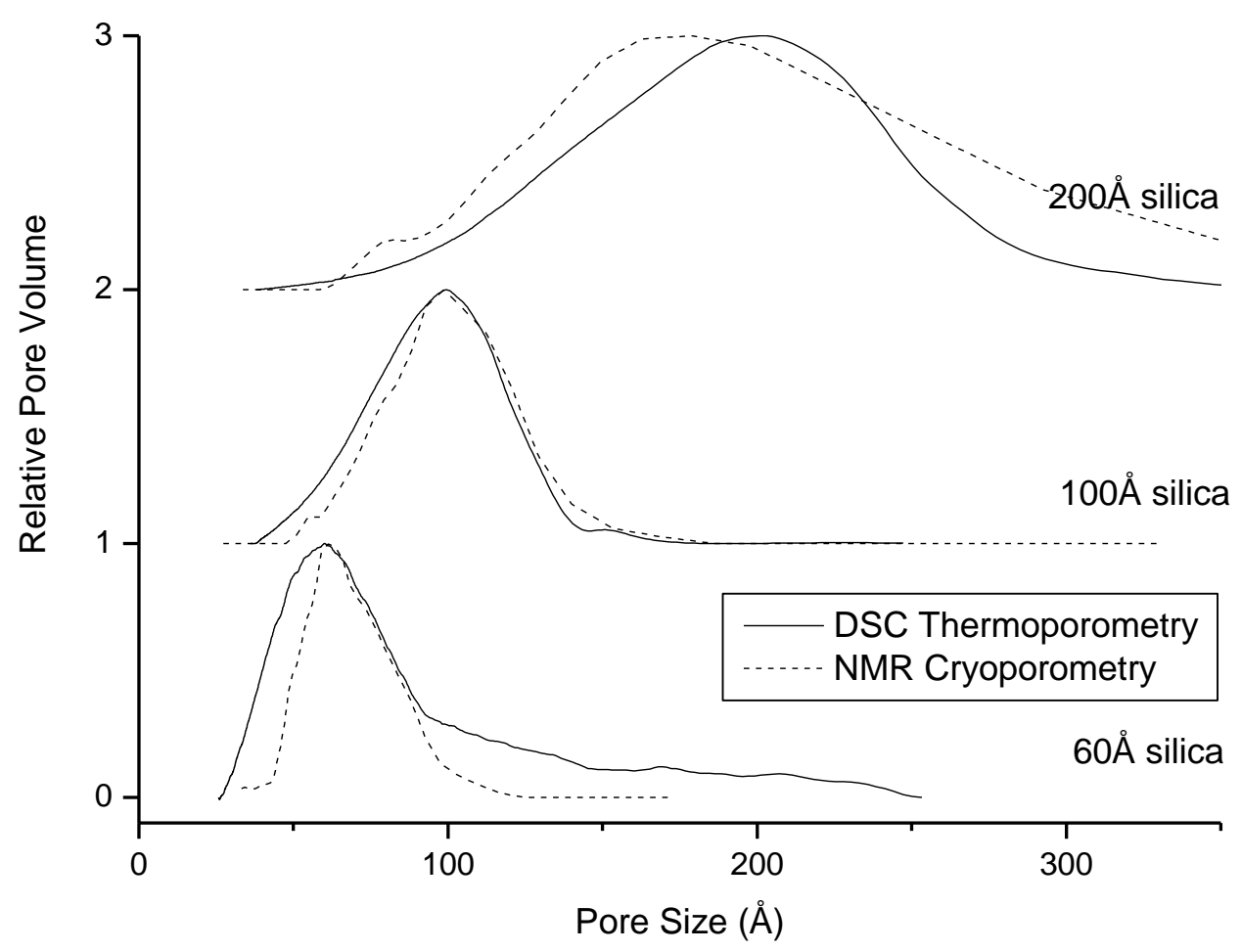

Fig 1(b) 


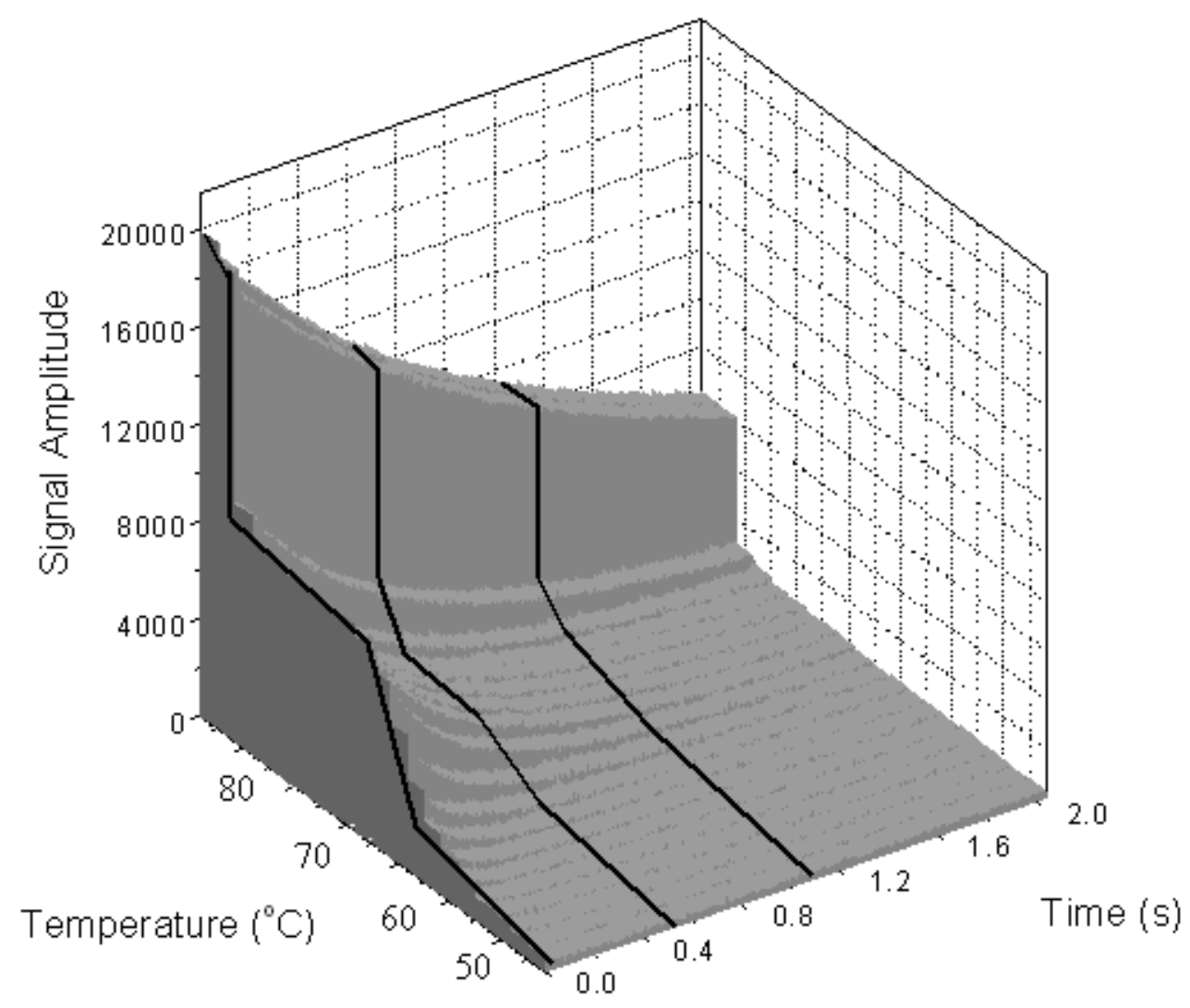

Fig 2(a)

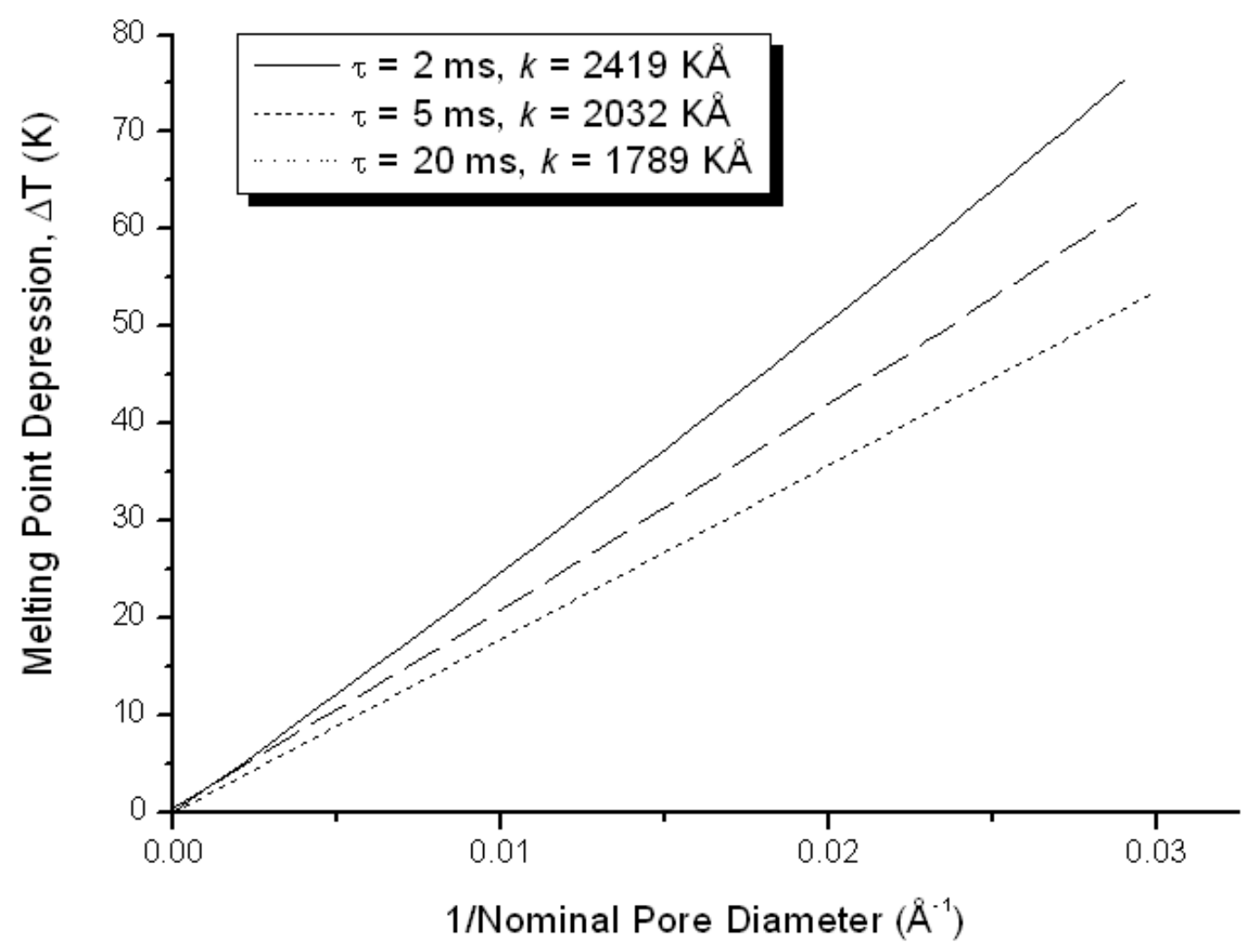

Fig 2(b) 

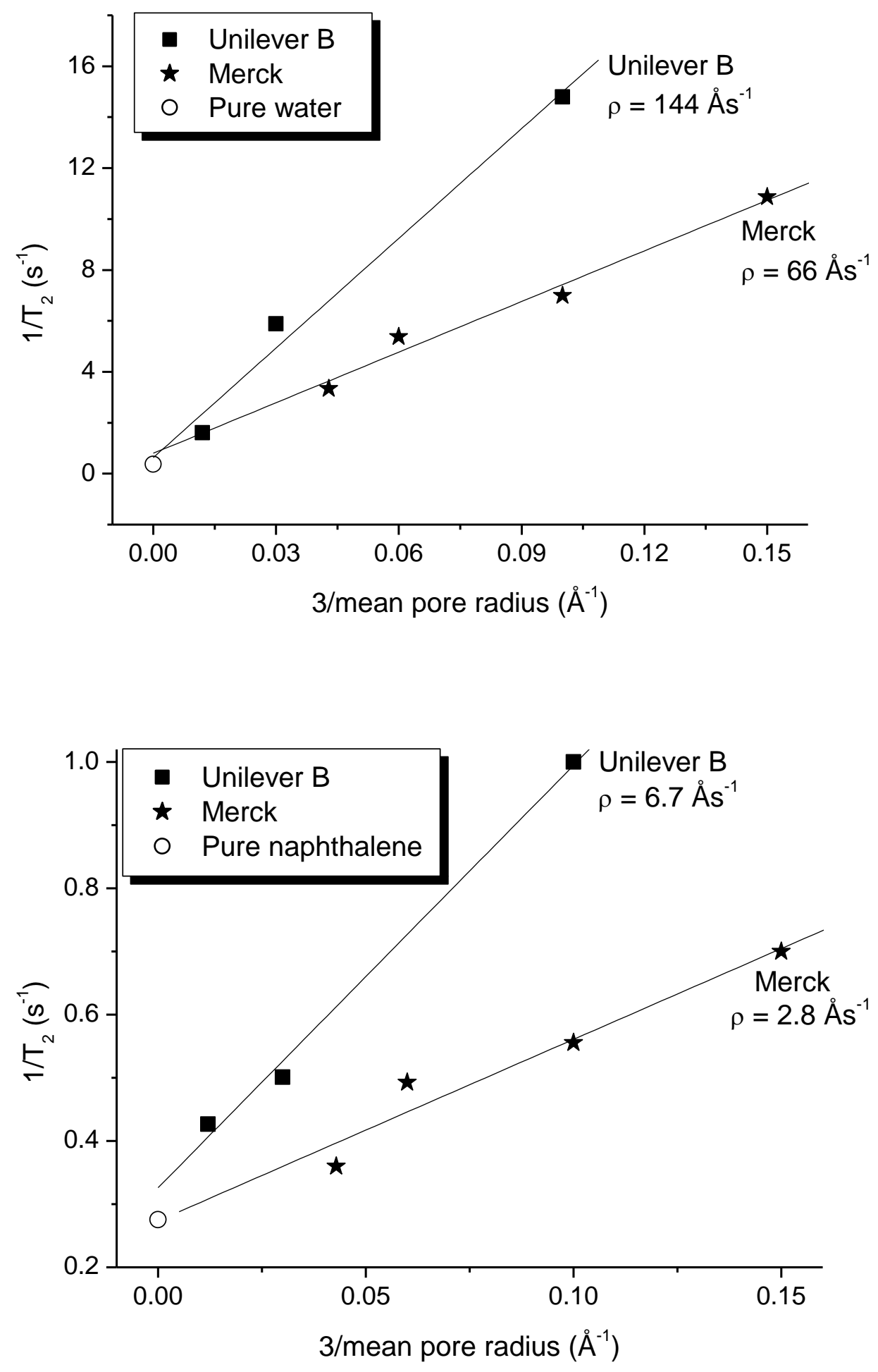

Fig 3 




Fig 4 